\title{
Immediate Breast Reconstruction
}

\author{
Chair: Elisabeth A. Kappos ${ }^{\mathrm{a}-\mathrm{c}}$ Walter P. Weber ${ }^{\mathrm{a}, \mathrm{c}}$ \\ Participants: Jana de Boniface ${ }^{\mathrm{d}, \mathrm{e}} \quad$ Yves Harder,g $^{\mathrm{f}}$ Jörg Heil ${ }^{\mathrm{h}}$ Florian Fitzal $^{\mathrm{i}}$ \\ Zoltan Matrai ${ }^{j}$ Oncoplastic Breast Consortium Study Group \\ a Breast Center, University Hospital Basel, Basel, Switzerland; 'b Department of Plastic, Reconstructive, Aesthetic \\ and Hand Surgery, University Hospital Basel, Basel, Switzerland; 'Faculty of Medicine, University of Basel, \\ Basel, Switzerland; ${ }^{d}$ Department of Molecular Medicine and Surgery, Karolinska Institutet, Stockholm, Sweden; \\ e Department of Surgery, Breast Unit, Capio St. Göran's Hospital, Stockholm, Sweden; ${ }^{f}$ Centro di Senologia della \\ Svizzera Italiana and Department of Plastic, Reconstructive and Aesthetic Surgery, Ospedale Regionale di Lugano, \\ Ente Ospedaliero Cantonale (EOC), Lugano, Switzerland; ${ }^{9}$ Faculty of Medicine, University of Zurich, Zurich,

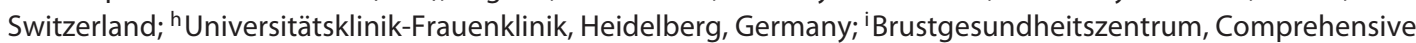 \\ Cancer Center Vienna, Abteilung für Chirurgie, Medizinische Universität Wien, Vienna, Austria; ${ }^{j}$ Department of \\ Breast and Sarcoma Surgery, Centre of Surgical Oncology, National Institute of Oncology, Budapest, Hungary
}

\section{Question 1: What Are Your Criteria to Choose 2-Stage over 1-Stage Implant-Based Breast Reconstruction?}

de Boniface: While 1-stage immediate breast reconstruction is my favoured option, I tend to avoid it in cases of large-volume reconstruction, necessitating expansion of skin and/or muscle, and in case of questionable quality of mastectomy skin flaps. In addition, other risk factors, such as smoking, a high BMI or other comorbidities, weigh towards a 2 -stage process, as does the prospect of post-mastectomy radiotherapy.

Harder:

- High probability to perform adjuvant radiotherapy of the "breast" after mastectomy (irradiation of thoracic wall, i.e., skin envelope, pectoralis muscles, and ribs).

- Critical perfusion of the skin envelope after skin-sparing mastectomy, nipple-sparing mastectomy or skinreducing mastectomy (clinical evaluation if ICG-fluorescent angiography is not available) that does not permit definitive reconstruction with adequate shaping of the mastectomy skin flaps around the definitive implant.
- Necessity to surgically treat a recently diagnosed breast cancer patient in which genetic testing is not possible/ cannot be completed before tumour surgery in due time, yet genetic mutation is probable and contralateral prophylactic mastectomy potentially indicated (i.e., BRCA 1, BRCA 2, p53, CHEK 2), and/or the patient has not decided upon the type of reconstruction.

- When the patient chooses to significantly augment the breast volume with regard to "prior mastectomy" ("if I have to sacrifice my breast, I'd rather have it augmented, because since then it was my desire to do so").

- Risk factors, such as smoking, ptotic and hypertrophic breast and high BMI, might be taken in consideration towards 2-stage reconstruction, yet ICG perfusion monitoring eases the decision-making on 1- and 2-stage reconstruction.

Heil: In cases were skin reduction is necessary for whatever reason (e.g., modified radical mastectomy), in patients with high-volume implant reconstructions $(>400 \mathrm{~mL})$, as well as in patients with high risk for wound healing complications (heavy smokers, diabetes, obesity, microangiopathy of any cause, etc.) I would favour 2- versus 1-stage implant-based breast reconstruction (IBBR). 
Fitzal: I use expander first in women who are active smokers or those who wish to have a larger breast size after reconstruction; otherwise, I do "direct to implant" in almost $80 \%$ of cases regardless of breast size, BMI or planned radiotherapy. In ptotic breasts, I almost always use a nipple full skin graft reconstruction. This ensures resection of as much breast tissue as possible behind the nipple. Moreover, data show that a nipple-areola complex used as a full skin flap has better sensations than other nipple-sparing procedures.

Matrai: Two-stage post-mastectomy breast reconstruction is the standard of care at my institution. Theoretically, I would perform 1-stage reconstruction if there is no indication for adjuvant radiotherapy, in small- or middle-volume breasts, in cases of good skin flap quality, if the patient wishes so and, lastly, if the affected breast has not been operated on before.

\section{Question 2: Which Type of Breast Reconstruction (Autologous/Implant-Based/Combination) Do You Favour in Case of Anticipated Post-Mastectomy Radiotherapy?}

de Boniface: I am not in favour of irradiating precious tissues, such as autologous flaps. Even though some centres report good results, I save autologous options for a later adjustment in case the patient is not satisfied with her implant reconstruction after radiotherapy. After detailed counselling, I use the implant as a potentially temporary solution, which in the vast majority of cases turns out to be the preferred long-term reconstructive strategy. It does give women time to think and to consider their alternatives before deciding on a larger autologous reconstruction, and, given lower complication rates, allows me to get them safely to their adjuvant treatment.

Harder: Temporary reconstruction with submuscular expander or prepectoral "babysitter" implant if adjuvant radiotherapy is highly probable or even clearly indicated to be preferred.

Definitive autologous reconstruction in case of highly probable or indicated adjuvant radiotherapy is not performed. Although the immediate complication rate is not increased in immediate reconstruction that gets irradiated, flap induration and shrinkage is very probable in the long term and, therefore, associated with loss of quality of life.

Definitive IBBR is performed in selected cases despite highly probable adjuvant radiotherapy, yet the patient is informed about the high risk of radiotherapy-induced capsular contracture that will probably result in corrective surgery, autologous reconstruction not to be excluded.
Heil: Delayed autologous reconstruction would be my first choice (subcutaneous implant at the time of mastectomy, followed by radiation, then autologous reconstruction).

Fitzal: For women with planned radiotherapy, I usually suggest directly performing implant-based reconstruction because I do not like to radiate autologous tissue. After radiotherapy to the implant-based reconstruction, autologous tissue may still be an option, and the patient has some more time to think about the procedure and the surgeons have time to plan everything without interfering with adjuvant therapy. If possible, I do not use an expander reconstruction in women with planned radiotherapy as morbidity of surgery after radiotherapy is higher. The increase in capsular fibrosis and contracture of a radiated implant has to be communicated. If it is necessary to use an expander (smoker), I try to rapidly expand and change it before radiotherapy (within 6-8 weeks after primary surgery). If radiotherapy has to be started immediately, I expand directly 1 week after final radiotherapy and exchange as fast as possible as the fibrosis and contraction effect of radiotherapy is usually seen later. I try to avoid bilateral immediate reconstruction for women with planned radiotherapy as the reconstructed healthy contralateral breast may be quiet in the way of the planned radiotherapy field.

Matrai: As primary treatment, I would favour nipplesparing mastectomy/skin-sparing mastectomy and subpectoral expander placement, and adjuvant radiotherapy, followed by autologous reconstruction, if possible. In case of anticipated major morbidity with free flap reconstruction, I would choose the latissimus dorsi flap as salvage procedure.

\section{Question 3: What Are Your Reasons to Favour Pre- over Subpectoral IBBR and What Percentage of Your Cases Is Done Prepectoral?}

de Boniface: Prepectoral reconstruction seems a more natural alternative, mitigating many of the drawbacks of submuscular implant placement. It allows a more natural feel, movement, clothing, and shape and probably causes less pain and preserves muscle function better. So, despite the significant lack of high-quality outcome data, most of my cases are prepectoral reconstructions, while I may revert to partly submuscular implant placement if doubting the quality of skin flaps in combination with larger implant size and comorbidities.

\section{Harder:}

- Pre- over subpectoral IBBR is preferred for prevention of breast animation, taking into consideration the risk 
of rippling, which seems (1) to be better tolerated by the patient than breast animation and (2) more easily corrected (e.g., autologous fat grafting).

- Of all IBBR, approximately 65 and 35\% are performed prepectoral and subpectoral, respectively.

Nowadays, the combination of expander, implant, acellular dermal matrix (ADM) and/or autologous fat grafting in multi-stage surgery allows to better "personalize" IBBR.

Heil: $80 \%$ of cases are done prepectoral; by favouring this position, we believe to reduce morbidity and create a more natural shape compared to subpectoral IBBR.

Fitzal: Almost all women are reconstructed with prepectoral IBBR. The reasons why I choose this type of reconstruction are:

- no breast animation,

- better breast shape,

- muscle may fill the upper pole unnaturally,

- muscle slips upwards and does not improve healing of the lower part of the skin, which is in fact the vulnerable part of the reconstruction regarding necrosis,

- muscle is destroyed and pain scores may be higher directly after surgery.

Most of these reasons, however, remain to be elucidated in prospective trials, such as the PREPEC OPBC-02 trial.

Matrai: Only about $10 \%$ of our cases are performed prepectoral, one of the reasons being that ADMs are not reimbursed. As plastic surgeon, I believe in the hypothesis to place implants into a well-vascularised tissue environment, resulting in more subpectoral reconstructions.

\section{Question 4: In Case of IBBR, When Do You Use a Mesh or ADM? Do You Have Any Preference in Material and Why?}

de Boniface: I nearly always use a mesh, either in the prepectoral space or as a hammock in partly subpectoral placement. I do, however, very much look forward to seeing more data on long-term outcome and optimal indications for using mesh or ADMs at all. As all material is procured via a tender process in my region, I exclusively use one type of a resorbable mesh (TIGR).

Harder: In case of prepectoral IBBR and thickness of the skin flap $\leq 1-1.5 \mathrm{~cm}$, a matrix is used to ideally cover the entire implant.

In case of subpectoral IBBR and independently of skin flap thickness, usually a mesh between the lower border of the pectoralis major muscle and the infra-mammary fold is used.
Currently, there are no preferences in material, as long as the matrix is meshed and the mesh fully absorbable.

Heil: Very seldom, I use TiLOOP Bra or TiLOOP Bra Pocket (if subcutaneous) or Tutomesh. I use TiLOOP in case of larger, anatomical implants to have less rotation probability, and Tutomesh in case of very, very thin patients.

Fitzal: I usually do not use any type of mesh or ADM, except in the case when the pocket is too large for the planned implant (e.g., TIGR, TiLOOP Bra Pocket); however, this is a rare case. I think that combining both the oncologic and the reconstructive surgery as a one-team approach ensures optimal planning and outcome, but this remains to be proven in trials. I do not see any advantage for ADM compared with mesh.

Matrai: I use ULTRAPRO because of the low price; I use other meshes only very rarely.

\section{Question 5: When Do You Favour Implant over Autologous Reconstruction and Why?}

de Boniface: I think, this is really a decision the patient needs to take together with me as a guide. Both strategies have their inherent advantages and disadvantages, and not all women are suitable for all options. So, once having discussed the pros and cons, I try to identify the patient's priorities, requirements and prerequisites, aiming to reach a decision together with her that mirrors more what she favours than what I favour. In therapeutic mastectomies with a patient who wishes to receive immediate reconstruction, however, I nearly always recommend implant-based reconstruction as laid out before, with the option of a later conversion to autologous reconstruction if desired. In case of previous radiotherapy, autologous alternatives may be the better option due to high complication rates and inferior quality of life after implant-based reconstruction - which is part of the counselling process.

Harder: In case of bilateral breast reconstruction without radiotherapy (i.e., adjuvant radiotherapy or planned radiotherapy), implant-based reconstruction is the first choice. Accordingly, it is the "least" invasive technique to reconstruct the breast in a symmetric way that may result in fairly high satisfaction despite foreign-body sensation, cold and firm breast and absence of donor site morbidity ("collateral damage"). It may give the patient some years of decent quality of life without flap surgery ("collateral damage"). In this respect, the best suitable patient is the one that decides to undergo bilateral prophylactic skinsparing mastectomy when diagnosed with gene mutation. 
Heil: Immediate IBBR is our standard of care in cases without radiation before or after mastectomy because of good aesthetic outcome, little morbidity and less morbidity (compared to autologous reconstruction).

Fitzal: The type of reconstruction depends on the patient's wish after clear informed consent. I think if softness is important to the patient, then autologous reconstruction has a benefit. If the cosmetic shape and volume are important for the patient and the woman wishes to have only minimal scars as well as a short surgical time, I opt for an implant (no data on level IV evidence). I regularly augment the other side, as unilateral implant-based reconstruction demonstrates significant asymmetry 5-10 years after surgery (level III evidence), which may not be seen after bilateral implant surgery (however, no data available on level IV evidence).

Matrai: I decide on an individual basis. Expander/implant-based reconstruction is the standard immediate breast reconstruction, if BMI, anatomy, skin quality, smoking habits and the vascular system allow it and the patient wishes it: TRAM (pedicled choice No. 1) or DIEP (choice No. 2). I use combination of latissimus dorsi flap plus implant only as a salvage procedure in case of skin necrosis after expander/implant-based reconstruction.

\section{Participants}

Assoc. Prof. Jana de Boniface

Department of Molecular Medicine and Surgery

Karolinska Institutet

Karolinska University Hospital, Solna, D2:02

17176 Stockholm, Sweden

jana.de-boniface@ki.se
Prof. Dr. med. Yves Harder

Ospedale Regionale di Lugano

Ente Ospedaliero Cantonale (EOC)

Via Tesserete 46, 6900 Lugano, Switzerland

yves.harder@eoc.ch

Prof. Dr. med. Jörg Heil

Universitätsklinikum Heidelberg

Universitätsklinik-Frauenklinik

Im Neuenheimer Feld 440

69120 Heidelberg, Germany

joerg.heilmed.uni-heidelberg.de

Prof. Dr. Florian Fitzal, MBA FEBS

Brustgesundheitszentrum

Comprehensive Cancer Center Vienna

21A Abteilung für Chirurgie

Medizinische Universität Wien, Währinger Gürtel 18-20

1090 Vienna, Austria

florian.fitzal@meduniwien.ac.at

Zoltan Matrai, MD, PhD, Dr. habil., F.E.B.S.

Department of Breast and Sarcoma Surgery

National Institute of Oncology

Rath Gy. u. 7-9.

1122 Budapest, Hungary

matraidok@oncol.hu

\section{Statement of Ethics}

This work has been completed according to the Declaration of Helsinki.

\section{Disclosure Statement}

The authors have no conflicts of interest to disclose. 